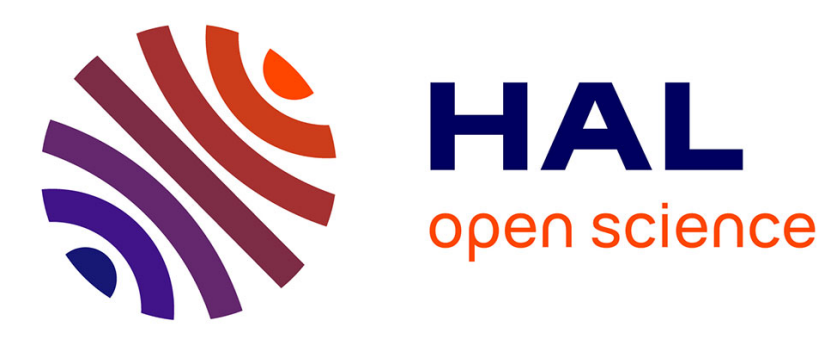

\title{
Far-and mid-infrared properties of carbon layers elaborated by plasma sputtering
}

Benoit Rousseau, Mohamed Ramzi Ammar, Denis Bormann, Patrick Simon, Hervé Rabat, Pascal Brault

\section{- To cite this version:}

Benoit Rousseau, Mohamed Ramzi Ammar, Denis Bormann, Patrick Simon, Hervé Rabat, et al.. Far-and mid-infrared properties of carbon layers elaborated by plasma sputtering. Applied Surface Science, 2016, 390, pp.1002-1008. 10.1016/j.apsusc.2016.07.165 . hal-01349935

\section{HAL Id: hal-01349935 https://hal.science/hal-01349935}

Submitted on 29 Jul 2016

HAL is a multi-disciplinary open access archive for the deposit and dissemination of scientific research documents, whether they are published or not. The documents may come from teaching and research institutions in France or abroad, or from public or private research centers.
L'archive ouverte pluridisciplinaire $\mathbf{H A L}$, est destinée au dépôt et à la diffusion de documents scientifiques de niveau recherche, publiés ou non, émanant des établissements d'enseignement et de recherche français ou étrangers, des laboratoires publics ou privés. 


\title{
Far-and mid-infrared properties of carbon layers
}

\section{elaborated by plasma sputtering}

\author{
Benoit Rousseau ${ }^{\mathrm{a} *}$, Mohamed Ramzi Ammar ${ }^{\mathrm{b}}$, Denis Bormann ${ }^{\mathrm{b} \dagger}$, Patrick Simon ${ }^{\mathrm{b}}$, Hervé \\ Rabat $^{\mathrm{c}}$, Pascal Brault ${ }^{\mathrm{c}}$
}

aLTN, UMR CNRS 6607, Site de la Chantrerie, 44402 Nantes Cedex

${ }^{\text {b} C E M H T I, ~ U P R ~ C N R S ~ 3079, ~ C S ~ 90055, ~} 45071$ Orléans Cedex 2, France

'GREMI, UMR CNRS 7344, 14, rue d'Issoudun, BP 6744, 45067 Orléans Cedex 2, France

Keywords : Carbon films, radiative properties, sputtering deposition, Infrared reflectivity, Raman spectroscopy

\begin{abstract}
The far-and mid-infrared reflectivity spectra of two carbon layers deposited on pure (100) silicon substrates by DC magnetron sputtering were investigated at room temperature in the 10$5000 \mathrm{~cm}^{-1}$ wavenumber range. Their structural and textural features were also studied by combining Raman spectroscopy, Field Emission Scanning Electron Microscopy (FESEM), High Resolution Transmission Electron Microscopy (HRTEM), X-Ray Reflectivity (XRR) and Rutherford Backscattering Spectroscopy (RBS). The set of results was used to discuss afterwards the influence of the texture on the infrared properties at varying length scale. Thereby, the two layers were found to be heterogeneous as assessed by RBS, XRR and FESEM and their thicknesses had been measured by XRR and FESEM. The information on the structural organization and "crystallite" size was given by Raman spectroscopy. The influence of both the textural and structural parameters on the measured infrared reflectivity spectra was discussed. Finally, a methodology was proposed to recover the intrinsic index of refraction and the intrinsic index of absorption of each layer.
\end{abstract}

\footnotetext{
${ }^{*}$ Corresponding author. Tel : +33 240683117. Fax + 33 240683141. E-mail address: benoit.rousseau@univ$\underline{\text { nantes.fr }}$ (Benoit. Rousseau)

${ }^{\dagger}$ This paper is dedicated to our colleague and co-author Denis Bormann, who passed away in 2009
} 


\section{INTRODUCTION}

The comprehensive knowledge of the thermal radiative properties of carbon-based materials is of crucial interest for calculating the amount of radiation fluxes exchanged within industrial systems in which they are integrated. Examples of carbon-based materials are thereby carbon foam for high-temperature thermal insulation [1], carbon/carbon composite used in atmosphere re-entry [2] and carbon-fibber-reinforced polymer for automotive and aircraft applications [3]. Several parameters can influence the thermal radiative properties (reflectance, transmittance, absorptance and emittance) [4] of these compounds such as the intrinsic ones, namely the structural state of the bulk parts (crystalline or amorphous) [5], and the extrinsic ones, namely their textures [6], and of course their thicknesses when they are shaped as a slab. From a radiative viewpoint, the term texture stands here for the spatial arrangement of the optical heterogeneities [7] such as the gaseous pores embedded in the carbon-based host matrix and their respective size distributions [8]. Let us remark that according to the thermal history undergone by the sample and to its texture anisotropy different radiative behaviours can be reached $[9,10]$. Moreover, the intrinsic radiative properties are also governed by the ratio of $s p^{2}$ (graphitelike) to $s p^{3}$ (diamondlike) chemical bonds [11].

For a sake of clarity, the intrinsic parameters governing the radiative properties are given by the corresponding complex index of refraction, $\widetilde{n}$, where $\widetilde{n}=n+i k$. In the previous expression $n$ is the index of refraction and $k$ is the index of absorption. For major industrial applications where carbon-based materials can be treated by infrared waves, the knowledge of the spectral dependence of $\widetilde{n}$ at any temperature is crucial either to predict their radiative properties or to solve heat transfer issues. However, it is well known that $\widetilde{n}$ exhibits a complex thermal dependence which is strongly connected to the structural state of the bulk part as explained more specially by Rouzaud et al. for carbon films [12] and Dombrovsky et $a l$. for carbon fibers [13]. Furthermore, let us notify that most of the reliable reported data concerning 
$\tilde{n}$ are mainly obtained for wavenumbers, $\sigma$, largely higher than those belonging to the infrared part of the electromagnetic spectra (typically for $\sigma>5000 \mathrm{~cm}^{-1}$, i.e., $0.8 \mathrm{eV}$ ) [14-16]. In fact, for the infrared spectral range, $\tilde{n}$ is often obtained by extrapolating, with more or less successes, appropriate analytical models used to interpret the optical spectra which are acquired at very high frequencies either by spectroscopic ellipsometry [17] and reflexion/transmission spectroscopy or by electron-energy-loss spectroscopy [14].

This brief overview shows that the precise determination of the spectral dependence of the thermal radiative properties of any given carbon-based materials is today precluded by a real lack of accurate values of theirs complex index of refraction, especially for the spectral range where the thermal radiation is relevant, i.e., the infrared spectral range. In order to solve this tricky problem for the infrared spectral range, one can propose to retrieve $\tilde{n}$ from infrared reflexion spectroscopy as it is classically performed for any dielectric and conducting oxides [18].

In the following, two carbon coatings elaborated with a plasma sputtering apparatus were studied in order to connect their far-and mid-infrared properties with both their structural and textural features. These films are currently used as electrodes for Proton Membrane Exchange Fuel Cell [19]. Note that the sputtering apparatus allows a rigorous control of the deposited quantities and textural features of the thin films.

To thoroughly describe both the structural and textural features of the two films, a combination of several techniques was used: Raman spectroscopy and High Resolution Transmission Electron Microscopy (HRTEM) for the structural state, Rutherford Backscattering Spectroscopy (RBS), X-Ray Reflectivity (XRR) and Field Emission Scanning Electron Microscopy (FESEM) for the texture. The data set is then confronted in order to propose a possible organization of the two carbon layers. According to this study, a procedure to retrieve the intrinsic complex index of refraction is exposed and some comments concerning 
the penetration length of the absorbed infrared light within fictitious layers with similar thicknesses are, after all, commented.

\section{EXPERIMENTAL SECTION}

Plasma sputtering deposition of carbon layers

A magnetron sputtering deposition system (MHS Equipment), with three independently biased DC planar magnetron targets, was used to sputter a graphite target (the two other targets are idle). The sputtering device is described in Ref. [19]. The resulting plasmas were well confined above the targets. The target discs of pure graphite (MCSE 99,9999\%) had a diameter of $10 \mathrm{~cm}$ and a thickness of $0.4 \mathrm{~cm}$. The target surfaces were $45^{\circ}$ tilted from the substrate surface direction, and the target surface centre was made to face the half radius of the substrate. The target centre was placed at a distance of $90 \mathrm{~mm}$ from the substrate (100) type silicon, $400 \mu \mathrm{m}$ thick). The residual base vacuum in the deposition chamber was better than $1 \times 10^{-7} \mathrm{~Pa}$ using a turbomolecular pump (ATM400, ALCATEL). A cold cathode/Pirani gauge (ACC 1009, ALCATEL) was used to measure the vacuum in the $10^{-7}-10^{5} \mathrm{~Pa}$ range. Prior performing any deposition, the target surface was sputter-cleaned for 3-5 min. The deposition was carried out at room temperature without extra heating or cooling of the substrate. Thus, the effective substrate temperature depended on the deposition conditions. The target voltage was fixed at $580 \mathrm{~V}$. The argon gas pressure $P_{A r}$ and flux $\Phi_{A r}$ were $0.2 \mathrm{~Pa}-20 \mathrm{sccm}$ (standard cubic centimeter per minute), and $2 \mathrm{~Pa}-80 \mathrm{sccm}$, leading to the elaboration of two samples. Increasing Argon pressure was expected to reduce density. Indeed, the collision number of sputter carbon atoms with the Argon atoms was increasing which in turn reduces the kinetic energy of carbon sputtered atoms. So less energy was transferred to the substrate, which reduced 
the possibility of atomic reorganisation in the growing film leading to lower film density. The deposition time was $30 \mathrm{~min}$.

\section{Experimental characterization}

The characterizations by Raman spectroscopy were performed in ambient conditions by using a Jobin Yvon T64000 spectrometer equipped with a charge-coupled device (CCD) camera (CEMHTI, Orléans, France) [20] working in single monochromator configuration with a Notch filter. All measurements were performed in the backscattering geometry. The excitation laser source was the $514.5 \mathrm{~nm}$ Ar line, with a power $(\sim 1 \mathrm{~mW})$ at the sample surface in order to avoid any degradation of the two layers. The beam was focused on the sample surface using a $\times 50$ objective, so that the diameter of the excited area is in the order of a few microns. .

A Jeol 2011 Transmission Electron Microscope (TEM) operating at $200 \mathrm{kV}$ (Laboratoire de Géologie, Paris, France) was used to investigate the nano-texture of each layer [21]. This TEM is equipped with a CCD camera (GATAN system ORIUS SC100, image size: 4008-2672 pixels). The High Resolution (HR) mode was used. For each layer, small fragments were smoothly extracted and deposited on a TEM copper grid, itself covered by a lace of amorphous carbon film. HRTEM images were then acquired on the very thin parts of the samples (thickness lower than $10 \mathrm{~nm}$ ) that lay across the carbon grid holes.

At higher length scale (micrometers), Rutherford Backscattering Spectroscopy (RBS) analysis of the porous films was performed with a Van de Graaf accelerator (CEMHTI, Orléans, France) using a $2 \mathrm{MeV}^{4} \mathrm{He}^{2+}$ ion beam. The diameter of the beam was about $1 \mathrm{~mm}$.

For X-ray reflectivity measurements, a Bruker D8 Discover apparatus operating at $\lambda_{C u_{K\langle\alpha\rangle}}=$ $1.5418 \AA$ (Institut Néel, Grenoble, France) was used. The monochromatization was obtained with a graded multilayer and then the beam was reduced in the reflectivity plane by 50 
micrometer- wide slits in order to minimize the irradiated footprint at the sample position. After careful adjustments of the sample, data were collected from 0 to 1 degree of incident angle with a $0.005^{\circ}$ step and corrected from irradiated area [22]. For data analysis, a Leptos ${ }^{\circledR}$ software based on Parratt formalism was used for X-ray reflectivity calculation.

To depict the micro-texture of each film, FESEM pictures were acquired with a Carl Zeiss SUPRA 40 device (GREMI, Orleans, France)

Finally, for even larger (millimetres) length scale, infrared reflectivity measurements were carried out in the $10-5000 \mathrm{~cm}^{-1}$ wavenumber spectral range oat $\mathrm{T}=300 \mathrm{~K}$ by using a Bruker IFS $113 \mathrm{~V}$ interferometer (CEMHTI, Orléans, France). An aluminium mirror was used as reference and the instrumental wavenumber resolution was set up at $4 \mathrm{~cm}^{-1}$. The spot diameter that was issued from the incident beam was $5 \mathrm{~mm}$. The interferometer had been described in details elsewhere [23].

\section{RESULTS AND DISCUSSION}

Texture of the two carbon layers

Two carbon layers were produced with a DC magnetron sputtering reactor as described in Ref. [19] . The two layers are respectively called S1 and S2, the acronym S corresponds here to sample. The sputtering parameters used for $\mathrm{S} 1$ and $\mathrm{S} 2$ were given in Table 1. Fig. 1 displays the FESEM images of the top view of S1 (a) and S2 (b) and of their respective cross-sections i.e. (c) and (d). From a thermal radiative viewpoint, both the carbon layers exhibit an heterogeneous texture since discontinuities can be observed on the cross-sections and a poral network can be guessed from the top-views. Thus, both the layers have a low surface roughness, this surface state resulting from the columnar structures with a mean lateral size of about 100 $\mathrm{nm}$ as it can be viewed on S1 (Fig. 1 (c)). Such columns are typical of the deposition process 
by plasma sputtering developed by some of the authors [24]. For S2, no columnar shape can be evidently seen due to the FESEM spatial resolution. At last, the thickness of $\mathrm{S} 1$ is found to be $700 \mathrm{~nm}$ whereas the thickness of $\mathrm{S} 2$ is found to be $200 \mathrm{~nm}$ as it can be assessed by the FESEM cross-sections of the two layers.

To get more quantitative information on the resulting average volumetric mass density of the two layers, XRR and RBS experiments were performed. X-ray reflectivity curves are shown in Figure 2. We reported also on the plot, as an indicative guideline, a simulation for bulk graphite with a volumetric mass density, $\rho_{g}$, of $2.28 \mathrm{~g} / \mathrm{cm}^{3}$. The low incident angle part corresponds to the total reflection regime. The reflectivity rapidly falls at the critical angle, $\theta_{c}$. This angle is related to the volumetric mass density of the material by the formula:

$$
\theta_{c}=\sqrt{(2 \delta)}
$$

where for X-ray, $\delta$ is the dispersion term of the complex index of refraction. Eq. (1) is true when the absorption term of the complex index of refraction can be neglected. Then $\delta$ can be written by the following relation:

$$
\delta=r_{e} \lambda^{2} \rho_{e} / 2 \pi
$$

where $\rho_{e}$ is the electron density, $r_{e}$ the classical radius of the electron and $\lambda$, the wavelength of X-ray.

From the measurements, it is evident that both films are less dense than bulk graphite and that between the carbon films the thinner one named S2 is the densest. Analysis using Leptos® software gives respectively for S1 and S2 a volumetric mass density, $\rho_{X R R}$, of $1.47 \pm 0.05 \mathrm{~g} / \mathrm{cm}^{3}$ and of $1.95 \pm 0.05 \mathrm{~g} / \mathrm{cm}^{3}$ (see table 2). 
The RBS analysis coupled with the SimNRA software [25] allows also the determination of the average volumetric mass of the two layers. Once the thickness of a given film, $h_{\text {FESEM }}$, is known, the RBS analysis can be used to give the value of the volumetric mass density, $\rho_{R B S}$, of the layer. Indeed the total number of atoms per $\mathrm{cm}^{2}, N_{S}$, is measured and when it is combined with the molar mass of the deposited specie, $M$ and the Avogadro number, $N_{A}$, it allows the calculation of $\rho_{R B S}$ :

$$
\rho_{R B S}=\frac{N_{S} M}{h_{F E S E M} N_{A}}
$$

However, according to the respective values of $h_{\text {FESEM }}$ deduced from the previous FESEM analysis for S1 and S2. Table 2 lists the two calculated values of the volumetric mass density. These two values are in pretty good agreement with those deduced from the analysis of the Xray reflectivity spectra. Thereby, it clearly confirms that $S 2$ is the densest film. Such a finding is consistent with the plasma sputtering deposition process since the lowest the argon pressure deposition is, the highest the kinetic energy of the sputtered carbon species is, making that the final deposit tends to be the more dense.

Structure of the two carbon layers

Fig.3 shows typical HRTEM images acquired respectively on small fragments extracted from S1 (Fig. 3a) and S2 (Fig. 3 b) films. The two images are typical of highly disordered graphitic structure nevertheless without becoming completely amorphous. Only small nanometer-sized fringes forming small Basic Structural Units (BSU) can be observed and these BSU are strongly disoriented. Qualitatively, it seems that the graphitic clusters of S2 are smaller 
than the ones of S1. In this work, even if HRTEM provides the direct imaging of the polyaromatic layers profile, this technique is less accurate to give quantitative information on the organisation degree. To go one step further, Raman spectroscopy was performed in order to quantitatively characterize the structural arrangements of the carbon atoms. Indeed, this optical technique is known to be powerful and the most sensitive to the full range of the structural states present in carbon-based materials, from perfectly crystalline to amorphous. Fig. 4 and 5 display the Raman spectra of the two carbons S1 and S2 in the first-order region (800-1900 $\left.\mathrm{cm}^{-1}\right)$. Their shapes classify these deposits in the amorphous carbon (a-C) category. The second order is not shown here since it is usually very small and broad for highly disordered carbons.

For sake of clarity, let us have a brief description of the structural characterization of carbons by Raman spectroscopy. Using the visible excitation, the Raman spectrum is dominated by the scattering of $\mathrm{sp}^{2}$-bonded graphitic carbons, because of their higher cross-section. Thus the clustering and disorder of the $\mathrm{sp}^{2}$ phase are the main factor affecting peak positions, width and intensity of the bands. These materials basically show common features in their Raman spectra in the first-order region. For highly disordered carbon, two broad bands appear at approximately $1600 \mathrm{~cm}^{-1}$ (the so-called $\mathrm{G}$ band) and at about $1350 \mathrm{~cm}^{-1}$ (the so-called D band). The $\mathrm{G}$ band is due to the bond stretching of all pairs of $\mathrm{sp}^{2}$ atoms in both rings and chains. The $\mathrm{D}$ band is due to the breathing modes of $\mathrm{sp}^{2}$ in rings and arises from a double resonant Raman process. However, another Raman band can be observed at $~ 980-1060 \mathrm{~cm}^{-1}$ using UV excitation. It can be attributed directly to $\mathrm{sp}^{3}$-bonded carbon (the so-called $\mathrm{T}$ band). One of the key parameters to monitor carbon bonding is the intensity ratio of the $\mathrm{D}$ and $\mathrm{G}$ peaks which represent a direct measure of the size, $L_{a}$, of the $\mathrm{sp}^{2}$ phase organised in rings "crystallite size". In amorphous carbons, Tuinstra-Koenig relation [26] is no longer valid and Ferrari and Robertson [11] assume that the intensity ratio $I_{D} / I_{G}$, using a Breit-Wigner-Fano (BWF) function for the $\mathrm{G}$ 
band and a Lorentzian shape for the $\mathrm{D}$ band, varies with the size of $\mathrm{sp}^{2}$-bonded sites according to the following relationship :

$$
I_{D} / I_{G}=C^{\prime}(\lambda) L_{a}^{2}
$$

with the constant value $C^{\prime}(514,5 \mathrm{~nm}) \approx 0.0055$. In this Ferrari-Robertson regime, the domain size varies as $I_{D} / I_{G}$, contrary to Tuinstra-Koenig regime. Figures 4 and 5 qualitatively show that the layer $\mathrm{S} 1$ has rather bigger aromatic domains than the layer $\mathrm{S} 2$ because $I_{D} / I_{G}$ is higher for S1. Qualitatively, the Full Width at Half Maximum (FWHM) of the G band is higher for S2 suggesting an increase of disorder for the polyaromatic layers.

According to the adopted fitting procedure, the parameters (band position, Full Width at Half Maximum) extracted from the fit are listed in Table 3 for S1 and in Table 4 for S2. Eq. (1) gives the typical size of the graphitic cluster which is found to be $8.3 \AA$ for $\mathrm{S} 2$, whereas the “crystallite size" is about $10.5 \AA$ for $\mathrm{S} 1$. This result is consistent with HRTEM observations. The other two key parameters to assess the disorder degree of carbon materials are the band displacement and the Full Width at Half Maximum of the G peak, $F W H M(G)$ [27]. Their values regarding the two samples are given in Tables 3 and 4 . One can note that for S1, $F W H M(G)$ is smaller and the position of the $\mathrm{G}$ band downshifts to lower frequencies compared to $\mathrm{S} 2$. These two evolutions suggest that $\mathrm{S} 2$ has a structure at nano scale slightly more disordered than the one of S1.

Infrared reflectivity spectra

The infrared spectra of the two samples are reported in Fig. 6. Qualitatively, the comparison of the shape of both the spectra suggests that the thickness of S1 layer is higher 
than the one of $\mathrm{S} 2$ by assuming that the two respective complex dielectric functions of the layers are not too different. Indeed the oscillations due to the interference fringes generated by the thin carbon layers are more tightened for the thickest one. This assumption is clearly confirmed by FESEM pictures on the respective cross-sections of the two layers (Fig. 1c and Fig. 1d) since the thickness of $\mathrm{S} 1$ is found to be $700 \mathrm{~nm}$ whereas the thickness of $\mathrm{S} 2$ is found to be $200 \mathrm{~nm}$.

The modelling of the infrared reflectivity spectrum of each film, $R(\omega)$, with an appropriate effective complex dielectric, $\widetilde{\varepsilon}_{\text {fim }}(\omega)$, can be judiciously used to determine, firstly, their exact effective complex index of refraction, $\tilde{n}_{\text {film }}(\omega)$. Here $\omega$ is the wavenumber expressed in $\mathrm{cm}^{-1}$ and $\widetilde{\varepsilon}_{\text {film }}(\omega)$ and $\tilde{n}_{\text {film }}(\omega)$ are a complex values.

Let us now detail the procedure used for recovering $\widetilde{\varepsilon}_{f i m}(\omega)$. In the case of a thin film deposited on an optically thick substrate $(1 \mathrm{~mm})$ and also optically polished on the contact side, one can express, $R_{F S}(\omega)$, as follows (Eqs. 5,6, and 7) :

$$
R_{F S}(\omega)=\left|\frac{\left.r_{a f}(\omega)+r_{f s}(\omega) e^{\left(-i 2 \omega \tilde{\varepsilon}_{f i l m}(\omega) d\right.} / c\right)}{\left.1+r_{a f}(\omega) r_{f s}(\omega) e^{\left(-i 2 \omega \tilde{\varepsilon}_{f i l m}(\omega) d\right.} / c\right)}\right|^{2}
$$

where $c$ is the speed of the light in vacuum,

$$
r_{a f}(\omega)=\frac{1-\sqrt{\widetilde{\varepsilon}_{f i m}(\omega)}}{1+\sqrt{\widetilde{\varepsilon}_{f i m}(\omega)}}
$$

is the air/film interface reflectivity and 


$$
r_{f s}(\omega)=\frac{\sqrt{\widetilde{\varepsilon}_{\text {film }}(\omega)}-\sqrt{\widetilde{\varepsilon}_{\text {sub }}(\omega)}}{\sqrt{\widetilde{\varepsilon}_{\text {film }}(\omega)}+\sqrt{\tilde{\varepsilon}_{\text {sub }}(\omega)}}
$$

is the film/substrate interface reflectivity. The complex dielectric function of the porous carbon layer is described as "effective" since it takes into account the average pore volume fraction, $f_{v}$ , and the pore shape as of the intrinsic complex dielectric function of the carbon species found in the layer. We assume in the following that $f_{v}$ is directly obtained from $d_{r}$ with $f_{v}=1-d_{r}$ where $d_{r}$ is the relative density given by $\rho_{\text {film }} / \rho_{g} \cdot \rho_{f i l m}$ is the volumetric mass density of one of the two films beforehand obtained by XRR or by RBS. Note that the intrinsic complex dielectric function of the carbon species is governed by the nano structure defining the layer, i.e., the size of the crystallized graphitic sheets as their degree of disorder. In Eq.7, $\widetilde{\varepsilon}_{\text {sub }}(\omega)$ is the complex dielectric function of the substrate. More details concerning the physical statement of Eq. 5 are given in Ref [4]. At this stage, three main parameters are required to model the experimental infrared reflectivity spectrum, namely $\widetilde{\varepsilon}_{\text {film }}(\omega), \widetilde{\varepsilon}_{\text {sub }}(\omega)$ and the film thickness, $d$ . For the case of the $\mathrm{Si}(100)$ substrate, it is easy to get its dielectric function, $\widetilde{\varepsilon}_{\text {sub }}(\omega)$ by the modeling its infrared reflectivity spectrum with a piecewise polynomial dielectric function model. The advantage of this model is that it does not require any knowledge of the microscopic mechanisms of absorption responsible for the infrared response. Of course, it had been shown for several materials that the shape of the dielectric function given by the piecewise polynomials dielectric function model is exactly the same as the one obtained by classical method (KramersKronig inversion) applied on dielectric compounds [28]. More details on this mathematical way of retrieving the optical function can be found in Ref. [28]. The same approach can be used to obtain $\widetilde{\varepsilon}_{\text {fim }}(\omega)$. For each layer, their respective thickness, $d$, is given by FESEM imaging. 
At least, the knowledge of $\widetilde{\varepsilon}_{\text {film }}(\omega)$ gives also access to its effective complex infrared conductivity, $\tilde{\sigma}_{\text {fim }}(\omega)$, using Maxwell 's equations since [18]:

$$
\mathfrak{R}\left(\tilde{\sigma}_{\text {film }}(\omega)\right)=\omega \varepsilon_{v} \Im\left(\widetilde{\varepsilon}_{\text {film }}(\omega)\right)
$$

In Eq. $8, \varepsilon_{v}$ is the dielectric constant of the vacuum, $\mathfrak{R}$ the symbol for the real part of a complex value and and $\mathfrak{I}$ the symbol for its imaginary part. Then, the static conductivity, $\sigma_{D C}$ is obtained by extrapolating at $\omega=0$ the value of the real part of the complex optical conductivity.

Note that main of the optical studies on carbonaceous thin films have been performed by spectroscopic ellipsometry [29] and/or by UV/visible reflectivity [30] for wavenumbers above $8000 \mathrm{~cm}^{-1} \sim 1 \mathrm{eV}$ higher than the ones of this work $\left(10<\omega<5000 \mathrm{~cm}^{-1}\right)$. Fig. 6 shows a good agreement, for both layers, between the experimental infrared reflectivity spectra and the simulated ones. For both layers, their thicknesses found thanks to the FESEM analysis, have been respectively used for modelling the infrared reflectivity of S1 and S2. In Fig. 7 is reported the real part of the effective optical conductivity of S1 and S2. The shapes of the curves are typical of a system where most of the electronic carriers are localized such as within semiconducting thin films $[18,31]$. For both the sample cases, two distinguished bands can be observed. For S1, the first band is active in the far infrared range (around $200 \mathrm{~cm}^{-1}$ ) and is mainly due to mobile carriers. The second band, localized in the mid infrared range, presents a more important spectral weight and is due to the localized electronic carriers. For S2, the first band appears more active in the far infrared whereas the second broad band is more developed in the near infrared range differently than for S1. By extrapolating the optical conductivity at zero frequency, S2 is found to be more conductive than $\mathrm{S} 1$ since $\sigma_{D C, S 2} / \sigma_{D C, S 1}=3.9$ on an 
electrical viewpoint. Since S1 is less dense than $\mathrm{S} 2\left(d_{r, S 1}=0.35\right.$ and $d_{r, S 2}=0.15$ by using XRR data), it indicates that the difference between the two electrical conductivities must be in reality lower. Moreover the S2 nano structure is more disordered than of S1 according to the HRTEM and Raman studies suggesting the probability rate for a trapped carrier to move from a graphitic cluster to another is higher. Some authors evoke a variable range hopping mechanism or thermally activated mechanism to explain the transport properties of this family of materials with covalent bonds [31]. [32].

By using the piecewise polynomial dielectric function model used for the retrieval of the effective complex dielectric functions of the two layers, now one can propose a method to compare their intrinsic complex refractive indexes, $\tilde{n}_{\text {film }}(\omega)$ since $\tilde{n}_{\text {fim ,int }}(\omega)=\sqrt{\widetilde{\varepsilon}_{\text {flim, int }}(\omega)}$. As recalled just before, if we assume that $f_{v}$ is directly given by $d_{r}$, then a complex dielectric function derived from the Effective Medium Theory and that takes into account $f_{v}, \widetilde{\varepsilon}_{\text {film,int }}(\omega)$ and an appropriate depolarisation factor [33] can be used to reproduce $R_{F S}(\omega)$. Such an approach is based on the hypothesis that the two carbons films of the present study may be assimilated as heterogeneous layers where nanometric void domains filled with air are embedded in a continuous matrix composed of disordered carbon.

By handling the Maxwell-Garnett model, one can obtain afterwards a useful relationship where $\widetilde{\varepsilon}_{\text {fim, int }}(\omega)=g\left(\widetilde{\varepsilon}_{\text {film }}(\omega), f_{v}\right)$ for the assumption where the porous domain may be rather spherical. The results for the intrinsic indexes of refraction, $n_{\text {film,int }}(\omega)$, are reported in Fig. 8 . They indicate a discrepancy between the intrinsic refraction indexes of the two layers suggesting that the reflective power of S1 is higher than the one of S2 in the far-and mid-infrared range. At $\omega=2000 \mathrm{~cm}^{-1}$, the extrinsic contribution due to the micro texture decreases the value of $\tilde{n}_{\text {fim }, \text { int S1 }}(\omega)$ of $37 \%$ whereas it is $11 \%$ for $\tilde{n}_{\text {fim }, S 2}(\omega)$. At higher wavenumber, at $\omega=4000$ $\mathrm{cm}^{-1}$, the diminution is $27 \%$ for $\tilde{n}_{\text {film,int,S1 }}(\omega)$ and $8 \%$ for $\tilde{n}_{f i m, \text { int }, S 2}(\omega)$. Furthermore, let us recall 
that the mean index of refraction of graphite is found to be near 2.6 [34] whereas the index of refraction of diamond is found to be 2.4. [35] Our calculated data appear as satisfactorily consistent with values available in the literature. On the other hand, in Fig. 9, the index of

absorption, $k_{\text {fim, int }, S 1}(\omega)$ of S1 is higher than the one of $S 2, k_{f i m, \text { int }, S 2}(\omega)$, in the mid-infrared range which involves, for example, that $\mathrm{S} 1$ is more efficient than $\mathrm{S} 2$ to totally absorb infrared light for fictive samples with identical thicknesses of $7.5 \mu \mathrm{m}$. In other words, fictitious films based on S1 have a mean transmittivity equal to 0 on the infrared spectral range when their thickness is higher than $7.5 \mu \mathrm{m}$ whereas films based on S2 have a mean transmittivity of 0.25 . This result, depending on the structural organisation of the disordered carbon domain, is very interesting when, for example, the study of the radiative properties of carbon fibers with similar diameter is addressed in the field of aeronautics.

This set of results shows therefore that it is possible to quantify the exact radiative contributions due to the structural features of the carbon domains, composing the two carbon layers of this work. To obtain such a result, we propose here to use an effective medium approach in order to dissociate the radiative contributions imposed ty the textural organizations of the two layers at the micro scale from their global radiative behaviours.

\section{CONCLUSION}

Both the effective and intrinsic infrared properties of two porous and disordered carbon layers have been investigated by infrared spectroscopy. The results show that the infrared properties are dependent on the protocol of elaboration used, i.e., magnetron sputtering in this work. The modification of the Argon pressure in the chamber of deposition has a direct consequence on the density of the carbon films. Then the radiative contribution imposed by the texture at micro scale and by the structural organisation of the carbon domains at the nano scale 
can be dissociated to recover the intrinsic complex refractive indexes of the two layers. Such a distinction is possible by assuming that the Effective Medium Theory is valid. Such a methodology lays on a thorough study based on Raman spectroscopy and HRTEM from the one side and XRR, RBS and FESEM from the other side. Raman spectroscopy and HRTEM give an outline on the disordered structural organisation of the carbon domains at the nano scale whereas XRR, RBS and FESEM allow the characterization of the textural properties at the micro scale. The combination of all these methods is necessary for understanding, afterwards, the precise role respectively played by the $\mathrm{sp}^{2}$ domains and by the average relative density of these of carbon-based films on their radiative properties. The methodology proposed in these work will provide a new insight on (i) the identification of the intrinsic optical properties when the micro-textured character of carbon film is important and on (ii) the prediction of the radiative properties of micrometric samples based on carbon, long as their intrinsic complex refractive indexes are known. 


\section{ACKNOWLEGEDMENT}

This work is supported by CAdD, FSE-EU, MHS Equipment, ACI Plasmapac and GDR-I CNRS "Plasmas". L. Ortega and P. Odier from Institut Néel (Grenoble, France) are acknowledged for x-ray reflectivity measurements. All authors would like to show gratitude to Y. Tessier from GREMI (Orléans, France), and to Dr. T. Sauvage from CEMHTI (Orléans, France). Finally, the authors thank Dr. J.-.N Rouzaud from the Geology Laboratory of Ecole Normale Supérieure (Paris, France) for helpful and stimulating discussions concerning the multi-scale organization of carbon films and for the MET study. 


\section{REFERENCES}

[1] D. Baillis, J. Sacadura, Thermal radiation properties of dispersed media: theoretical prediction and experimental characterization, 67 (2000) 327-363.

[2] J. Lachaud, N. Bertrand, G. Vignoles, G. Bourget, F. Rebillat, P. Weisbecker, A theoretical/experimental approach to the intrinsic oxidation reactivities of $\mathrm{C} / \mathrm{C}$ composites and of their components, Carbon, 45 (2007) 2768-2776.

[3] W. Brandl, G. Marginean, V. Chirila, W. Warschewski, Production and characterisation of vapour grown carbon fiber/polypropylene composites, Carbon, 42 (2004) 5-9.

[4] R. Siegel, J.R. Howell, Thermal Radiation Heat Transfer 4 edition ed., Taylor \& Francis, 2001.

[5] A. Sadezky, H. Muckenhuber, H. Grothe, R. Niessner, U. Pöschl, Raman microspectroscopy of soot and related carbonaceous materials: spectral analysis and structural information, Carbon, 43 (2005) 1731-1742.

[6] B. Rousseau, D. De Sousa Meneses, P. Echegut, J.-F. Thovert, Textural parameters influencing the radiative properties of a semitransparent porous media, International Journal of Thermal Sciences, 50 (2011) 178-186.

[7] J. Yin, L. Pilon, Efficiency factors and radiation characteristics of spherical scatterers in an absorbing medium, JOSA A, 23 (2006) 2784-2796.

[8] B. Rousseau, D. De Sousa Meneses, P. Echegut, J.F. Thovert, Textural parameters influencing the radiative properties of a semitransparent porous media, International Journal of Thermal Sciences, 50 (2011) 178-186.

[9] K. Lafdi, S. Bonnamy, A. Oberlin, Textures and structures in heterogeneous pitch-based carbon fibres (asspun, oxidized, carbonized, and graphitized), Carbon, 31 (1993) 29-34.

[10] J. Rouzaud, A. Oberlin, Structure, microtexture, and optical properties of anthracene and saccharose-based carbons, Carbon, 27 (1989) 517-529.

[11] A.C. Ferrari, J. Robertson, Interpretation of Raman spectra of disordered and amorphous carbon, Physical Review B, 61 (2000) 14095-14107.

[12] J. Rouzaud, A. Oberlin, C. Beny-Bassez, Carbon films: structure and microtexture (optical and electron microscopy, Raman spectroscopy), Thin Solid Films, 105 (1983) 75-96.

[13] L.A. Dombrovsky, D. Baillis, Thermal Radiation in Disperse Systems: An Engineering Approach, Begell House New York, 2010.

[14] A.B. Djurisic, E.H. Li, Optical properties of graphite, Journal of Applied Physics, 85 (1999) 7404-7410.

[15] N. Savvides, Optical constants and associated functions of metastable diamondlike amorphous carbon films in the energy range 0.5-7.3 eV, Journal of Applied Physics, 59 (1986) 4133-4145. 
[16] S. Gupta, B. Weiner, G. Morell, Ex situ spectroscopic ellipsometry and Raman spectroscopy investigations of chemical vapor deposited sulfur incorporated nanocrystalline carbon thin films, Journal of Applied Physics, 92 (2002) 5457.

[17] J. Hong, A. Granier, A. Goullet, G. Turban, In situ deposition and etching process of aC: H: N films in a dual electron cyclotron resonance-radio frequency plasma, Diamond and Related Materials, 9 (2000) 573-576.

[18] F. Gervais, Optical conductivity of oxides, Materials Science and Engineering: R: Reports, 39 (2002) $29-92$.

[19] H. Rabat, C. Andreazza, P. Brault, A. Caillard, F. Béguin, C. Charles, R. Boswell, Carbon/platinum nanotextured films produced by plasma sputtering, Carbon, 47 (2009) 209-214.

[20] T. Gries, L. Vandenbulcke, P. Simon, A. Canizares, Stresses in textured and polycrystalline cubic films by Raman spectroscopy: Application to diamond, Journal of Applied Physics, 102 (2007) 083519.

[21] M.R. Ammar, J.N. Rouzaud, C. Vaudey, N. Toulhoat, N. Moncoffre, Characterization of graphite implanted with chlorine ions using combined Raman microspectrometry and transmission electron microscopy on thin sections prepared by focused ion beam, Carbon, 48 (2010) 1244-1251.

[22] A. Gibaud, S. Hazra, C. Sella, P. Laffez, A. Désert, A. Naudon, G. Van Tendeloo, Particle layering in the ceramic-metal thin film Pt-Al_\{2\} O_ $\{3\}$, Physical Review B, 63 (2001) 193407.

[23] F. Gervais, Infrared and Millimeter Waves, Vol, 8 (1983) 279-339.

[24] H. Rabat, P. Brault, Plasma Sputtering Deposition of PEMFC Porous Carbon Platinum Electrodes, Fuel Cells, 8 (2008) 81-86

[25] M. Mayer, SIMNRA, a simulation program for the analysis of NRA, RBS and ERDA, in: J.L. Duggan, I.L. Morgan (Eds.) 15th International Conference on the Application of Accelerators in Research and Industry, American Institute of Physics, 1999, pp. 541.

[26] F. Tuinstra, J. Koenig, Characterization of graphite fiber surfaces with Raman spectroscopy, Journal of Composite Materials, 4 (1970) 492-499.

[27] M.R. Ammar, J.N. Rouzaud, How to obtain a reliable structural characterization of polished graphitized carbons by Raman microspectroscopy, Journal of Raman Spectroscopy, 43 (2012) 207-211.

[28] D. De Sousa Meneses, B. Rousseau, P. Echegut, P. Simon, Retrieval of Linear Optical Functions from Finite Range Spectra, Applied spectroscopy, 61 (2007) 1390-1397.

[29] S. Gupta, B.R. Weiner, G. Morell, Ex situ spectroscopic ellipsometry investigations of chemical vapor deposited nanocomposite carbon thin films, Thin Solid Films, 455-456 (2004) 422-428. 
[30] F.W. Smith, Optical constants of a hydrogenated amorphous carbon film, Journal of Applied Physics, 55 (1984) 764-771.

[31] C. Godet, Variable range hopping revisited: the case of an exponential distribution of localized states, Journal of Non-Crystalline Solids, 299-302, Part 1 (2002) 333-338.

[32] A. Tibrewala, E. Peiner, R. Bandorf, S. Biehl, H. Lüthje, Transport and optical properties of amorphous carbon and hydrogenated amorphous carbon films, Applied surface science, 252 (2006) 5387-5390.

[33] D.E. Aspnes, Optical properties of thin films, Thin solid films, 89 (1982) 249-262.

[34] M. Bruna, S. Borini, Optical constants of graphene layers in the visible range, Applied Physics Letters, 94 (2009) 031901.

[35] D.F. Edwards, E. Ochoa, Infrared refractive index of diamond, J. Opt. Soc. Am., 71 (1981) 607-608. 


\section{TABLE CATIONS}

TAB. 1: Experimental parameters for elaboration of $\mathrm{S} 1$ and $\mathrm{S} 2$ by magnetron sputtering. $U_{\text {Target }}, I_{\text {Target }}, P_{\text {Target }}$ are the carbon target bias voltage $(\mathrm{V})$, discharge current (A) and applied power $(\mathrm{W})$, respectively. $t$ is the deposition time, $P_{A r}$ is the Argon pressure in reactor and $\left\langle E_{c}\right\rangle$ is the mean kinetic energy of the sputtered carbon atoms at the substrate location.

TAB. 2: Textural parameters of S1 and S2: height by SEM and XRR, volumetric mass density by XRR and RBS

TAB. 3: Intensity and FHWM of the Breit-Wigner-Fano and of the Lorentzian functions used to fit the Raman spectrum of S1

TAB. 4: Intensity and FHWM of the Breit-Wigner-Fano and of the Lorentzian functions used to fit the Raman spectrum of S2 
Tab. 1

\begin{tabular}{ccccccc}
\hline Sample & $\begin{array}{c}U_{\text {Target }} \\
(\mathrm{V})\end{array}$ & $I_{\text {Target }}(\mathrm{A})$ & $\begin{array}{c}P_{\text {Target }} \\
(\mathrm{W})\end{array}$ & $t(\mathrm{mn})$ & $P_{A r}(\mu \mathrm{bar})$ & $\left\langle E_{c}\right\rangle(\mathrm{eV})$ \\
\hline $\mathrm{S} 1$ & 580 & 2.30 & 1330 & 30 & 20.0 & 0.04 \\
\hline $\mathrm{S} 2$ & 580 & 0.74 & 430 & 30 & 2.2 & 4.5 \\
\hline
\end{tabular}


Tab. 2

\begin{tabular}{ccccc}
\hline Sample & $\begin{array}{c}\mathrm{h}_{\text {SEM }} \\
(\mathrm{nm})\end{array}$ & $\begin{array}{l}\mathrm{h}_{\text {XRR }} \\
(\mathrm{nm})\end{array}$ & $\begin{array}{c}\rho_{X R R} \\
\left(\mathrm{~g} / \mathrm{cm}^{3}\right)\end{array}$ & $\begin{array}{c}\rho_{R B S} \\
\left(\mathrm{~g} / \mathrm{cm}^{3}\right)\end{array}$ \\
\hline & & & & \\
S1 & $700 \pm 5$ & $700 \pm 5$ & $1.47 \pm 0.05$ & $1.59 \pm 0.05$ \\
S2 & $200 \pm 4$ & $190 \pm 4$ & $1.95 \pm 0.05$ & $2.08 \pm 0.05$ \\
\hline
\end{tabular}


Tab. 3

\begin{tabular}{cccc}
\hline $\begin{array}{c}\text { Band } \\
\text { position } \\
\left(\mathrm{cm}^{-1}\right)\end{array}$ & Intensity & $\begin{array}{c}\text { FHWM } \\
\left(\mathrm{cm}^{-1}\right)\end{array}$ & Band \\
\hline 1349 & 2463 & 239 & $\mathrm{D}$ \\
1576 & 4091 & 156 & $\mathrm{G}$ \\
\hline
\end{tabular}


Tab. 4

\begin{tabular}{cccc}
\hline $\begin{array}{c}\text { Band } \\
\text { position } \\
\left(\mathrm{cm}^{-1}\right)\end{array}$ & Intensity & $\begin{array}{c}\text { FHWM } \\
\left(\mathrm{cm}^{-1}\right)\end{array}$ & Band \\
\hline 1352 & 3736 & 281 & $\mathrm{D}$ \\
1535 & 9789 & 254 & $\mathrm{G}$ \\
\hline
\end{tabular}




\section{FIGURE CAPTIONS}

Fig. 1 : FESEM pictures of the two carbon layers (a) top view of $\mathrm{S} 1$; (b) top view of S2; (c) cross-section of S1; (d) cross-section of S2.

Fig. 2 : XRR measurements of S1 and S2 corrected from irradiated footprint area. The long dashed curve corresponds to S1 whereas the continue line correspond to S2. The dashed curve is a simulation for a bulk graphite surface. The sharp change of the slope corresponds to the critical angle for total reflection and is related to the volumetric mass density of the material. The bumps observed at $0.44^{\circ}$ are due to the critical angle of the silicon substrate.

Fig. 3 : HRTEM images of S1 (a) and S2 (b). Each nanometer-sized black fringe corresponds here to a given Basic Structural Unit (BSU)

Fig. 4 : Raman spectrum of S1

Fig. 5 : Raman spectrum of S2

Fig. 6 : Infrared reflectivity spectra of S1 (top part) and S2 (bottom part) from 10 to $5000 \mathrm{~cm}^{-1}$ acquired at room temperature. For both samples, the thin curve corresponds respectively to the experimental spectrum and the symbols are relative to the simulated spectrum.

Fig. 7 : Real part of the complex effective optical conductivity at $\mathrm{T}=300 \mathrm{~K}$ of $\mathrm{S} 1$ (red open circle) and S2 (black open square) obtained by a piecewise polynomial dielectric function model in the far and the mid-infrared region. 
Fig. 8 : Real part of the complex index of refraction of S1 and S2 at T $=300 \mathrm{~K}$. The effective index of refraction are represented by thin lines and the intrinsic index of refraction by thick lines.

Fig.9 : Imaginary part of the complex index of refraction of S1 and S2 at T = 300 K. The effective index of absorption are represented by thin lines and the intrinsic index of absorption by thick lines. 

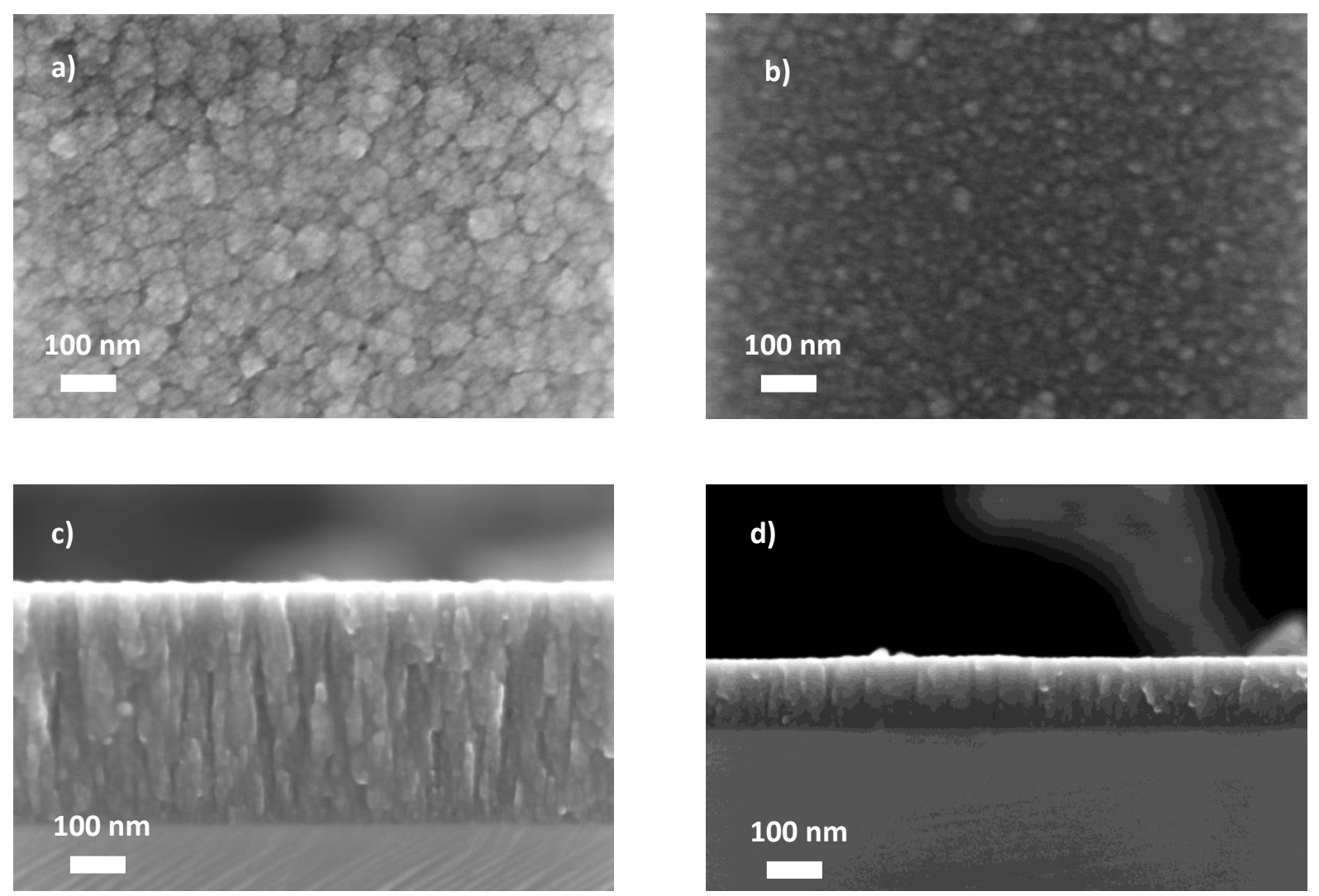

Fig. 1 


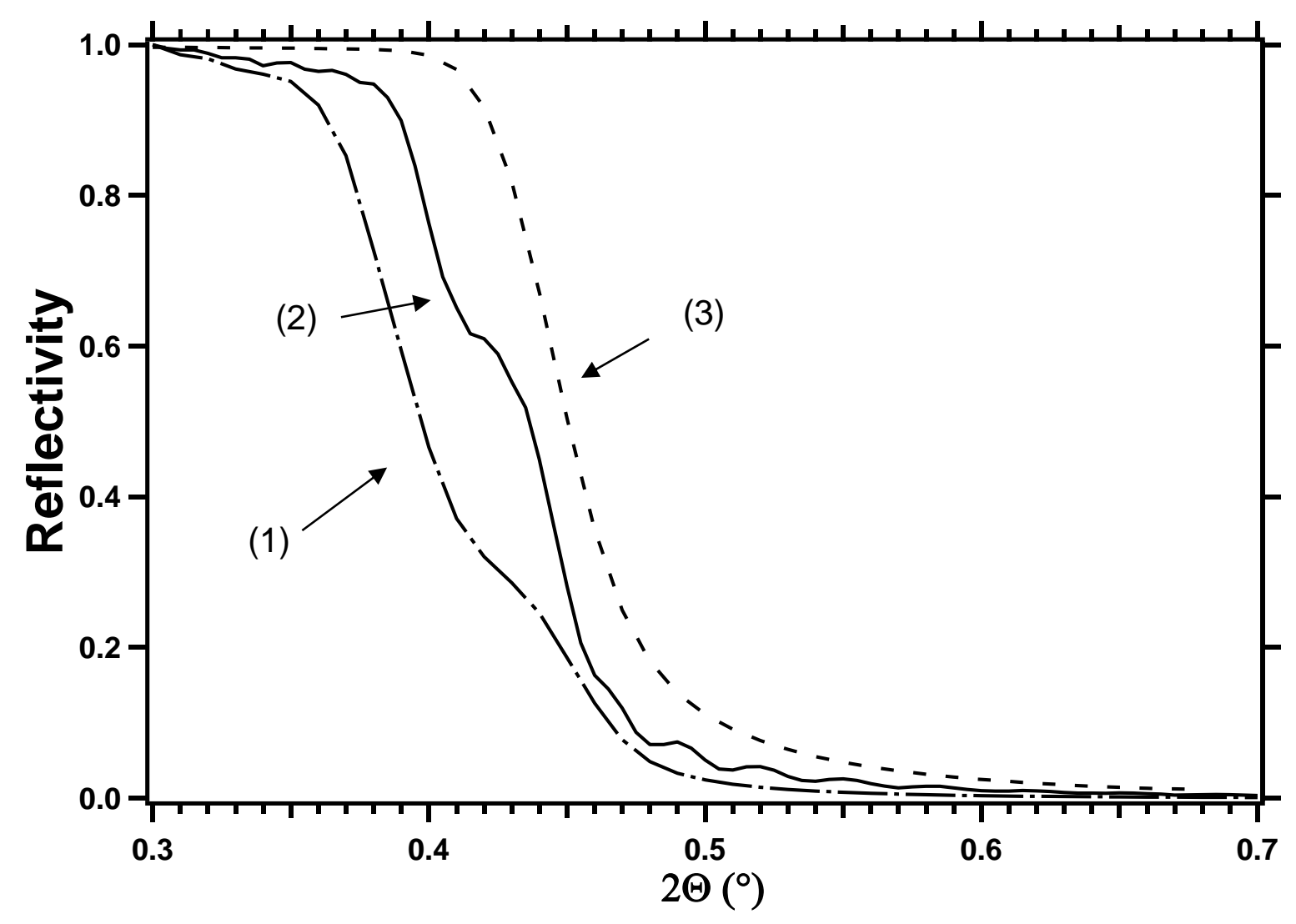

Fig. 2 

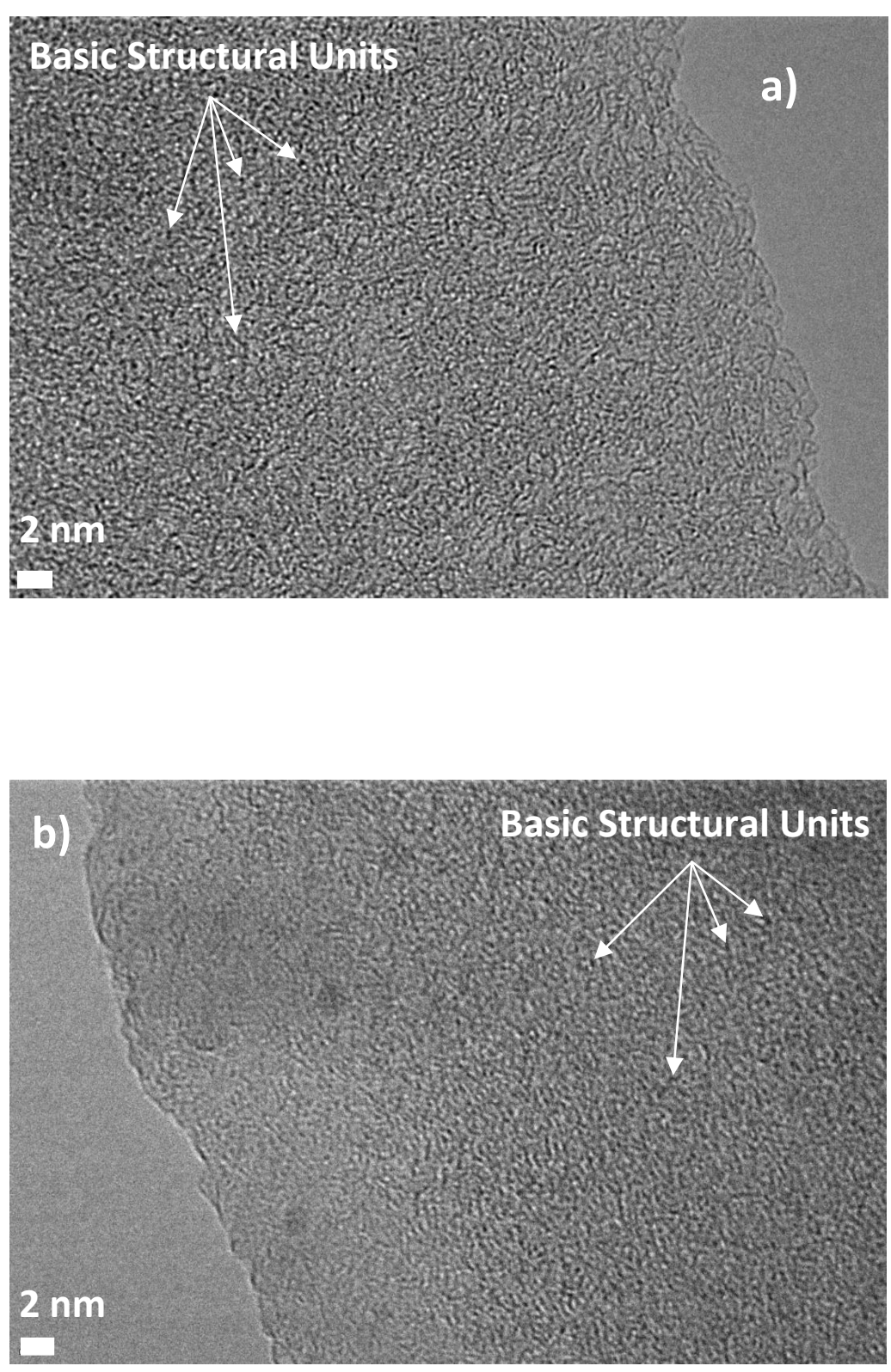

Fig. 3 


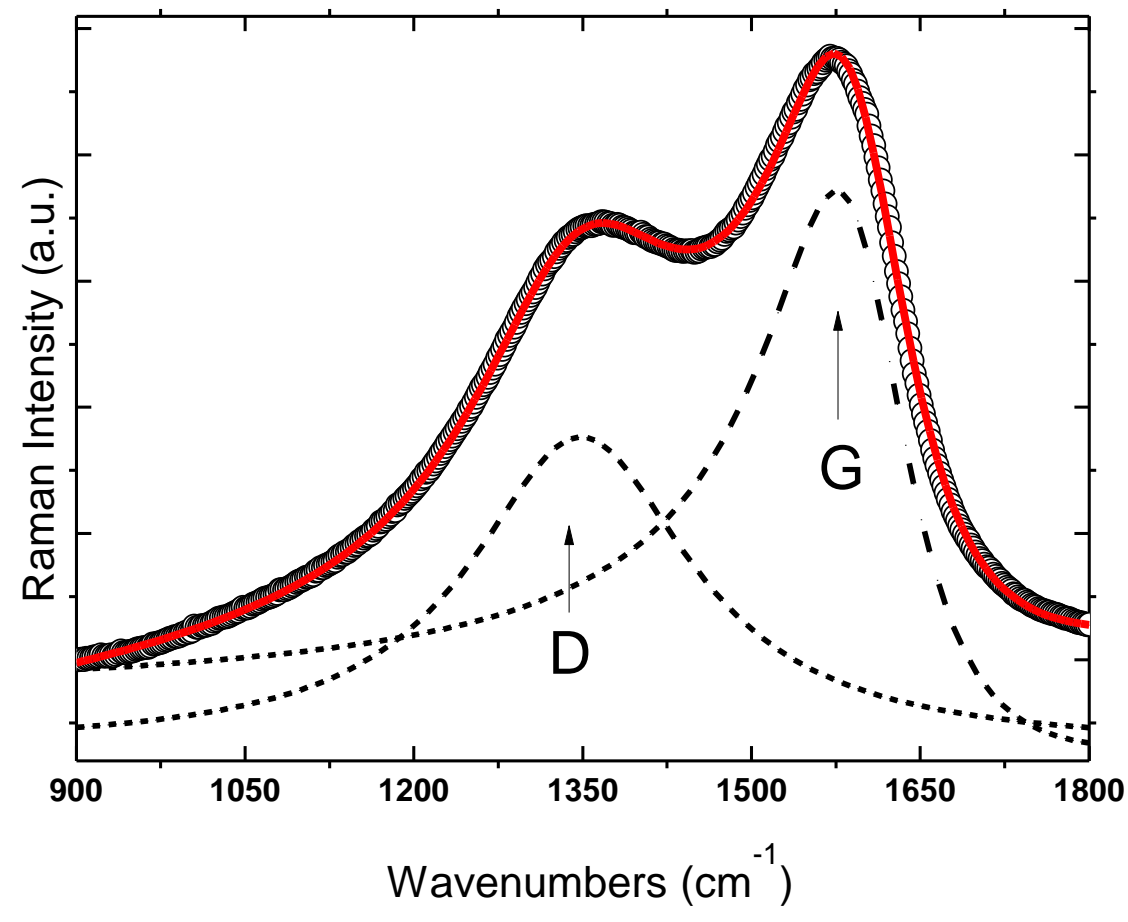

Fig. 4 


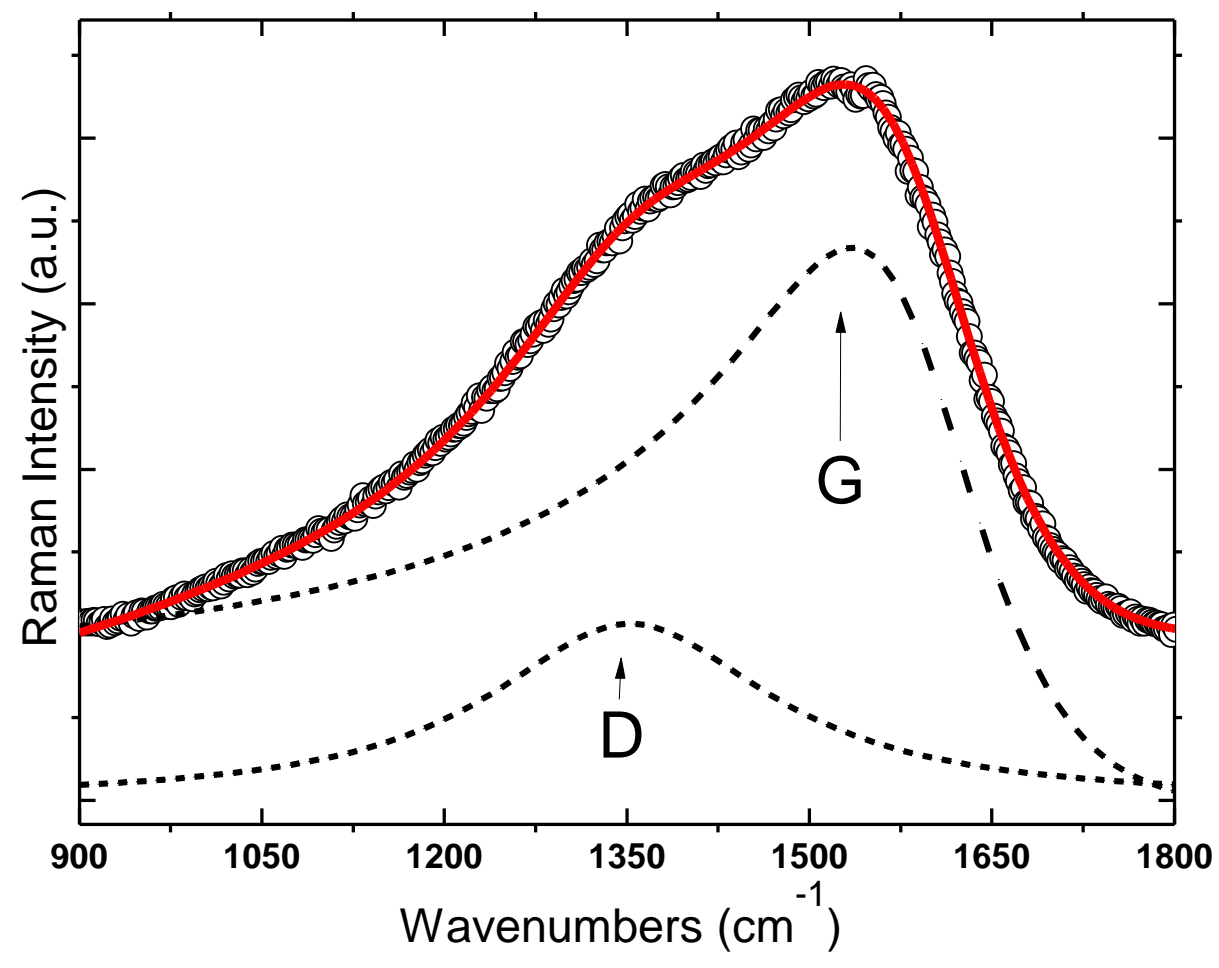

Fig. 5. 


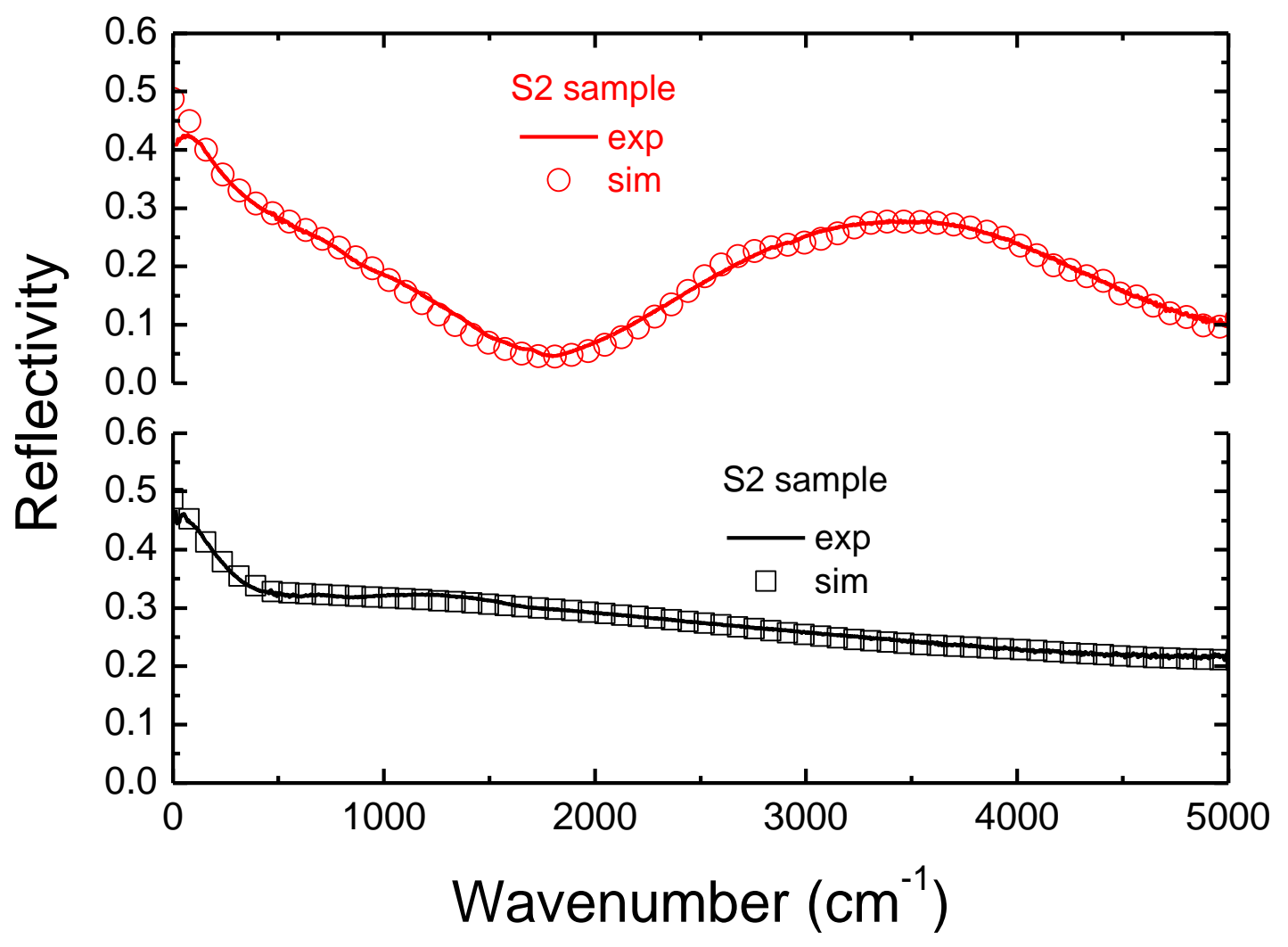

Fig. 6. 


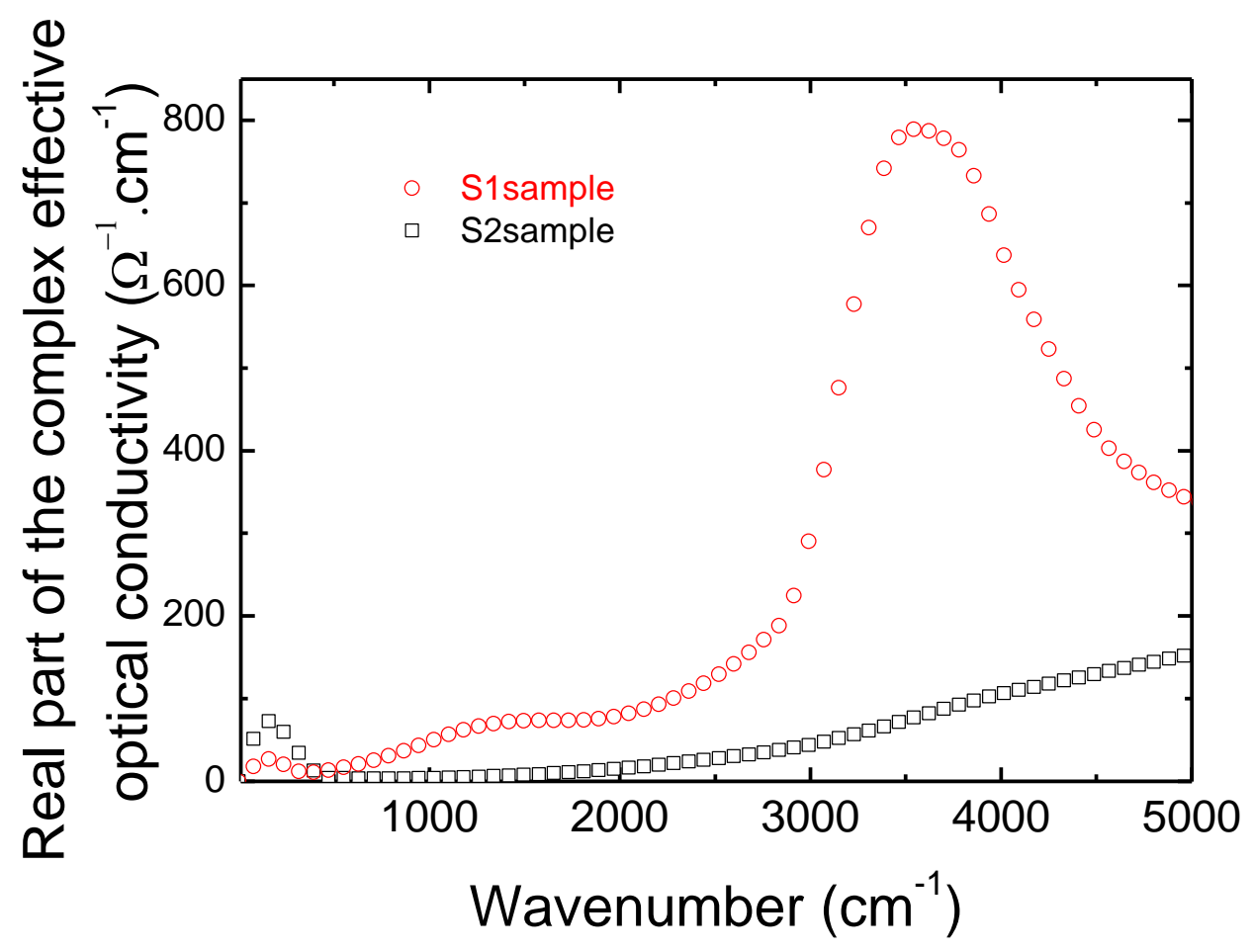

Fig. 7 


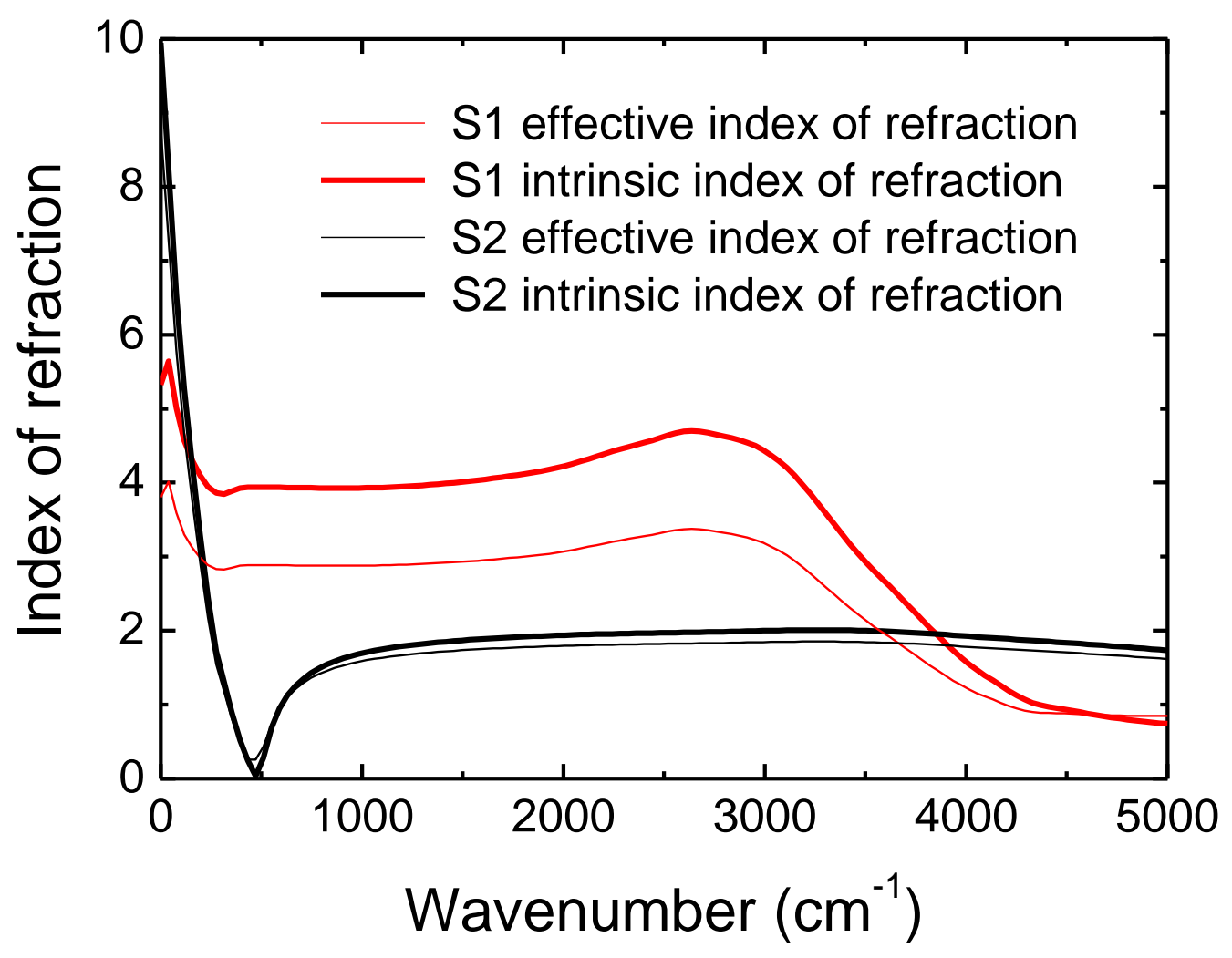

Fig. 8 


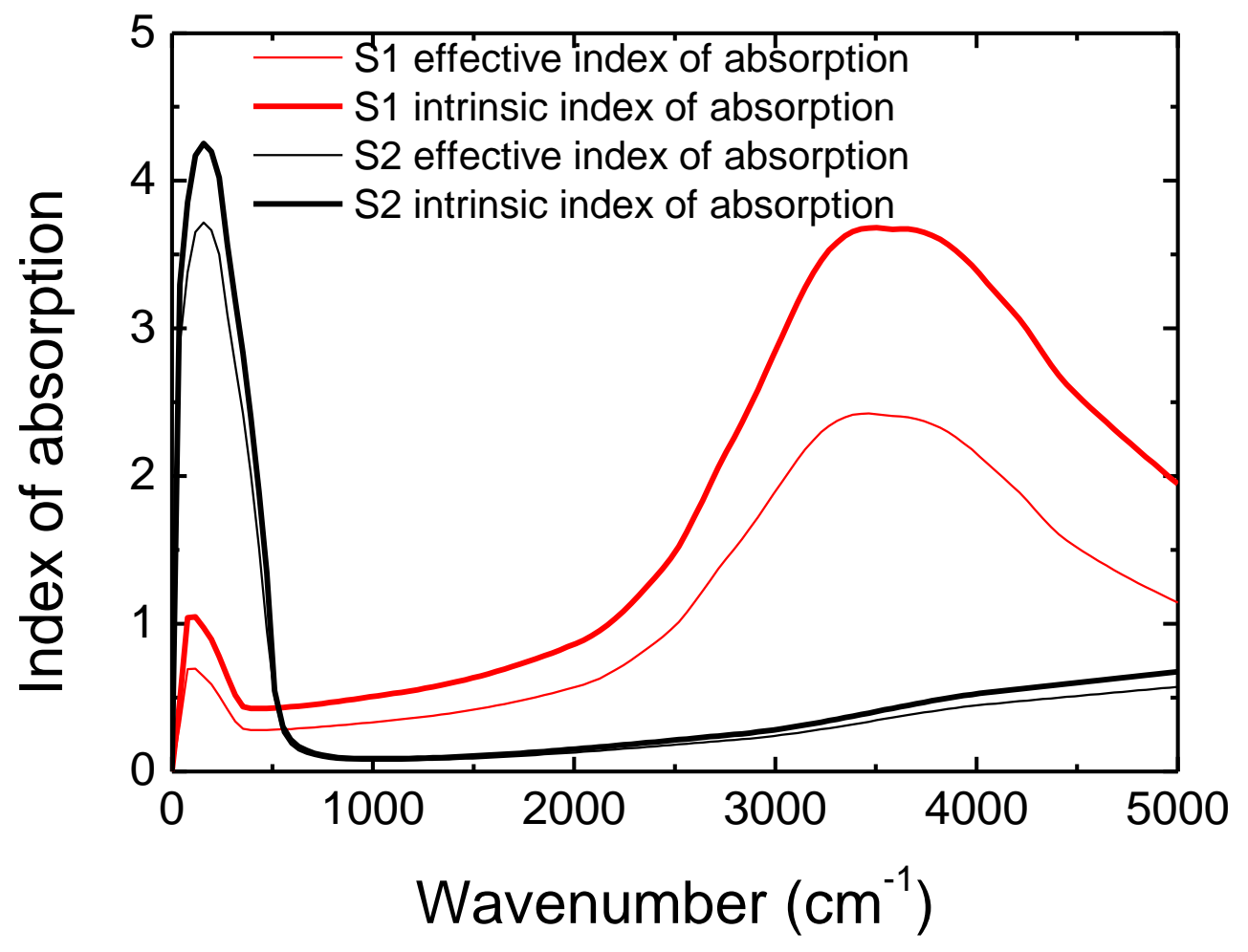

Fig. 9 